\title{
Pressure sores prevention for paraplegic people: Effects of visual, auditory and tactile supplementations on overpressures distribution in seated posture
}

\author{
Olivier Chenu ${ }^{\mathrm{a}, \mathrm{b}, *}$, Yohan Payan ${ }^{\mathrm{a}}$, P. Hlavackova ${ }^{\mathrm{a}, \mathrm{c}}$, Jacques Demongeot $^{\mathrm{a}}$, Francis Cannard ${ }^{\mathrm{b}}$, \\ Bruno Diot ${ }^{\mathrm{b}}$ and Nicolas Vuillerme ${ }^{\mathrm{a}}$ \\ ${ }^{a}$ TIMC-IMAG Laboratory, CNRS UMR, Grenoble, France \\ ${ }^{\mathrm{b}}$ IDS-SA, Montceau-les-mines, France \\ ${ }^{\mathrm{c}}$ Palacky University, Olomouc, Czech Republic
}

\begin{abstract}
This paper presents a study on the usage of different informative modalities as biofeedbacks of a perceptual supplementation device aiming at reducing overpressure at the buttock area. Visual, audio and lingual electrotactile modalities are analysed and compared with a non-biofeedback session. In conclusion, sensory modalities have a positive and equal effect, but they are not equally judged in terms of comfort and disturbance with some other activities.
\end{abstract}

Keywords: Pressure sores, prevention, perceptual supplementation, sensory substitution, tactile

\section{Introduction}

A pressure sore is a localized tissue injury often yielded by a mechanical compression of these tissues between bones and external surfaces [1]. Pressure sores treatments can be very long and difficult, thereof they are dramatic for the victims and expansive for the society [2]. Pressure sores are frequent in people with disabilities, such as diabetics or quadri- or paraplegics, mainly because of their loss (or default) of somatosensory perceptions. It is indeed paresthesia

${ }^{*}$ Corresponding author: Olivier Chenu, TIMC-IMAG Laboratory, CNRS UMR 5525, Grenoble, France. E-mail: olivier.chenu@ imag.fr. (sensory perception induced by a lack of oxygen in the organic tissues) that allows an able-bodied person releasing regularly overpressures applied onto his/her skin. In the particular case of paraplegia, pressure sores are mainly formed onto the ischias and the sacrum [3]. Paraplegic people are indeed very often in seated postures because of their motor disability, and do not feel the paresthesia signals from their buttock.

The principle of sensory substitution, or perceptual supplementation, stipulates that a lost or deficient sensory modality can be supplied by the mean of another intact sensory modality [4].

Thereof, we hypothesised in a recent article than providing an artificial perception of the painful informations as paresthesia could help neurologically 
impaired people to avoid pressure sores [5]. This was made by the mean of a perceptual supplementation device that consists in supplying paresthesia by a tactile modality. The components of this perceptual supplementation device were: (1) a pressure sensors map, (2) an lingual electrotactile actuator and (3) a laptop that activates the actuator depending on the data collected on the sensors, begging the user to move in some direction. The results have shown that able-bodied people could use such a perceptual supplementation device to release overpressures accumulated for a while.

However, lingual electrotactile perception is not usual at all as a sensory modality. Along these lines, a more usual sense (like visual or auditory feedbacks) could be more accurate in terms of perception and efficiency. Indeed, the electro-tactile stimulations could be neglected while having a concurrent perceptual/attentional recreative activity. Besides, in order to largely develop a pressure sores prevention system, it has to be accepted by the potential users.

To answer these questions, we designed the present study to compare the efficiency and acceptability of a perceptual supplementation device, equipped with the lingual electrotactile modality, against more usual sensory modalities: a visual and an auditory one.

\section{Materials and methods}

\subsection{Subjects}

Eight able-bodied people and one paraplegic person voluntarily participated to this study, by giving their written consent.

\subsection{Materials}

The perceptual supplementation devices are composed of three parts:

(1) a sensor part that can acquire the data from the lost/deficient modality that the device will supply;

(2) an actuator part that can stimulate the modality chosen as the new vector of perception; and

(3) a coupling part that collects the data from the sensor part, treats and transforms them in order to activate the actuators.
Our pressure sore prevention device is aimed to supply paresthesia perceptions by collecting buttock pressures. Thereof, this device consists in:

(1) a pressure sensors map system, at the skin/seat interface;

(2) different actuators for various modalities are used: a laptop screen for the visual feedback, a stereo headphone for the auditory one and a Tongue Display Unit [5] for the lingual electrotactile modality; and

(3) a laptop coupling the sensors and the actuator.

\subsubsection{Sensor}

We used the Vista Medical pressure mapping system. This device is a flexible pressure map applied at the seat/skin interface. A matrix of 1024 pressure sensors $(32 \times 32)$ are regularly spread on a surface of $45 \times 45 \mathrm{~cm}$. Each sensor can measure a pressure between 0 and $200 \mathrm{mmHg}$ with a precision lower than $1 \mathrm{mmHg}$. This is guaranteed by a system of calibration that is provided with the map. The map allows a capture of the whole matrix at a frequency of $5 \mathrm{~Hz}$.

\subsubsection{Coupling}

A laptop is used to collect the pressure data from the sensors and to activate the different actuators. This point is described in the Methods section. Also, this laptop is used to display a movie that the subjects are asked to watch during the experiment.

\subsubsection{Actuator}

Three kinds of actuators will be tested in this experiment (see Fig. 1).

(1) Tactile actuator: the Tongue Display Unit (TDU) is a lingual electrotactile device. Previously developed by Paul Bach-y-Rita et al. [6], the TDU consisted in a matrix of 144 electrodes $(12 \times 12)$ put in contact with the antero-superior part of the tongue. Each electrode can convey an electrical signal. This TDU was first designed for visual substitution. To adapt this device to health problematic, the TIMC-IMAG Laboratory developed his own wireless TDU $[7,8]$, consisting in a matrix of 36 electrodes $(6 \times 6)$ put in contact with the tongue. Each electrode can lead independently an AC electrical signal 

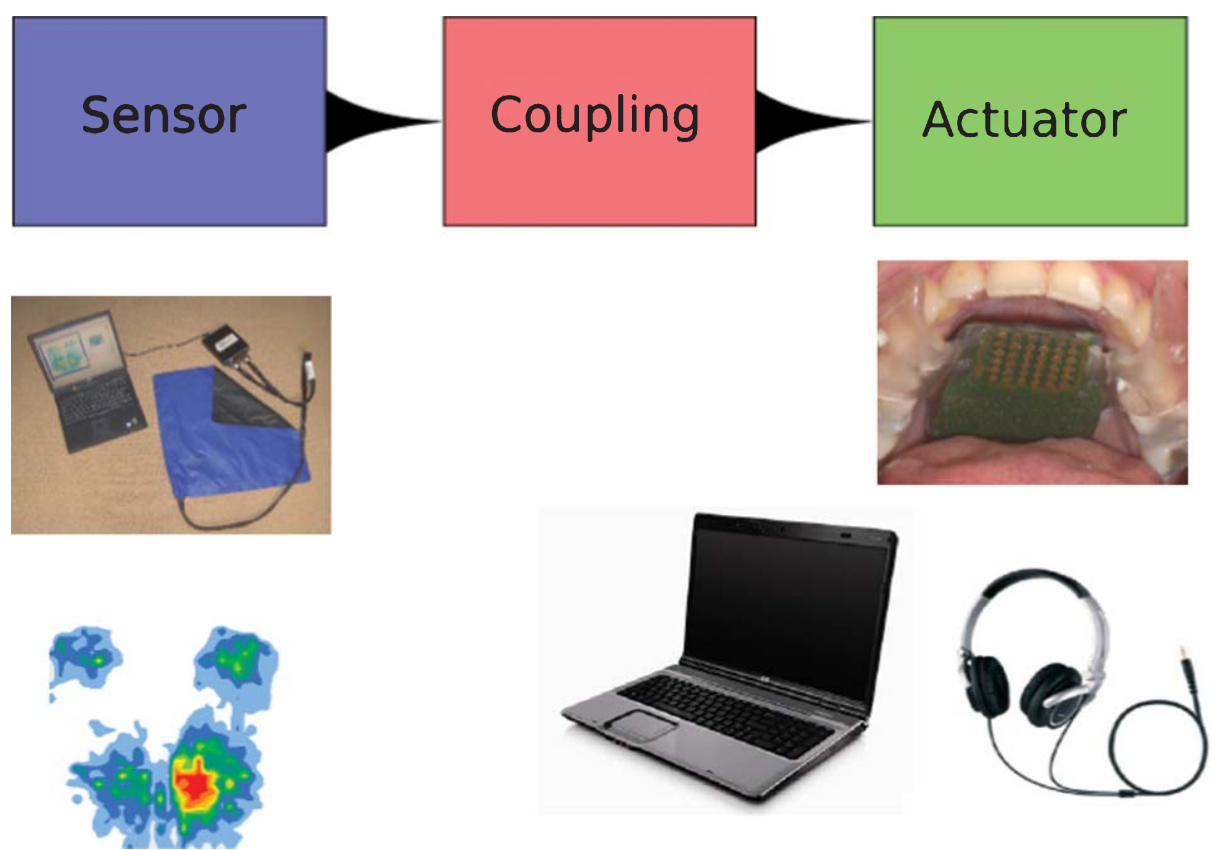

Fig. 1. Global scheme of the perceptual supplementation system. The pressure map forms the sensor part, the laptop screen, the headphones or the TDU form the actuator part, and the coupling part is an algorithm implemented in the laptop.

between 0 and 5 Volts. The whole matrix can be refreshed at a frequency of $3 \mathrm{~Hz}$.

(2) Visual actuator: the left and right sides of the laptop screen are used to convey the visual biofeedback signal.

(3) Auditory actuator: a $1 \mathrm{kHz}$ sinusoidal signal displayed in a stereo headphones is used for the auditory modality.

\subsection{Method}

\subsubsection{Calibration of the TDU}

The inter-subjects sensitivity to electrical stimulations on the tongue is very variable [9]. Thereof, before each experiment, the maximum voltage for each subject was calibrated. The subject had the TDU inside his/her mouth with no stimulation. For each pattern of stimulation used in this study (see Fig. 2 for a pattern example), the pattern was displayed with a null intensity (so that the user could not feel it). Then, the voltage was slowly increased until the subject felt a high but not painful stimulation.

Once the calibration phase was ended, the subject had to recognize easily each pattern of stimulation to be sure he/she did perceive correctly the different
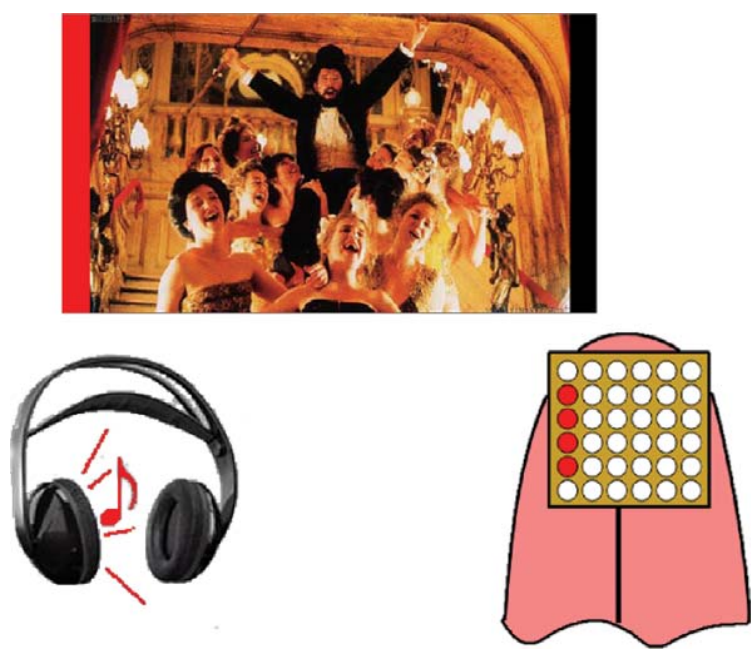

Fig. 2. The different actuator conveying the same message "left"; this message takes the form of the side of the screen becoming red for the visual feedback, a superimposed sound in the left headphones for the auditory feedback, and the activation of four electrodes at the left side of the TDU matrix for the tactile feedback.

stimulations. To do this, the experimenter applied randomly one of the patterns at the intensity decided during the previous calibration session, and the subject 
had to make a sign to indicate the direction of the stimulation he/she felt. After 6 correct answers, this pretest was ended. The subjects had the possibility to change the calibrated intensities during the period of test.

\subsubsection{Reference postures and pressure maps}

Before the experiment, subjects were asked to seat on the centre of the map and to lean towards the left and right directions. For each of these three distinct Reference Postures (left, centre and right), pressure maps were acquired and stored in a database as, respectively, RP_L, RP_C and RP_R.

\subsubsection{Concurrent task and equipment}

While having an attentional activity, paraplegic persons are estimated by the clinicians to be highly risky for pressure sores formations. These persons focus indeed on their task and may forget to mobilize themselves, particularly if they do not have the perception of paresthesia anymore.

It was therefore chosen to ask subjects to watch a movie during the experimental session. They were seated on the pressure map, watching a film displayed on the laptop screen, wearing headphones with the movie soundtrack, and the TDU was put inside their mouth.

\subsubsection{During the experiment}

Whatever the actuator that was chosen for the experiment, if subjects did not perceive any sensory stimulations coming from the actuator, they can seat as they want; they could freely adapt their posture. At some moments, signals were sent to the actuator, indicating a directional postural change that should relieve the measured overpressures. These signals were chosen to be very simple in order to induce left or right displacements of the subject chest. A "left" signal (i.e. the lightening of the left part of the laptop screen, a signal sent to the left headphone or stimulations of the left TDU electrodes) was supposed to mean a "pain" due to an overpressure in the left part of the buttocks. Subjects had therefore to move their chest towards the opposite direction (i.e. right) of the perceived signal. This kind of biofeedback was chosen since we wanted the signal to represent a danger, as the paresthesia information. This signal lasted for 10 seconds maximum, or less if the subjects reached a safe posture before the end.

The signal took the form of a side of the laptop screen becoming red, a $1 \mathrm{kHz}$ sound superimposed to the movie soundtrack or the activation of side electrodes of the TDU (see Fig. 2).

Each subject did execute four experimental sessions: a control one in which there was no stimulation, and three biofeedback sessions (visual, auditory and electrotactile conditions).

The chronological orders of the experimental sessions were randomized for each subject, so that the eventual effect of fatigue is negligible. Each session lasted for 7 minutes. In the biofeedback conditions, stimulations were sent every 20 seconds $\pm 4 \mathrm{~s}$ for a total of 20 stimulations per session. Between two sessions, subjects were allowed to stand up and to take a break if they wished.

\subsubsection{Algorithm decision: How are biofeedback signal generated?}

The coupling algorithm implemented in the laptop aims at analysing in real time the overpressures and at sending regularly the suitable biofeedback signal.

When a stimulation is about to be sent, the algorithm operates in 3 successive steps: (1) estimation of the current posture of the subject, (2) calculation of the posture that is the most suitable to reduce cumulated overpressures, and (3) activation of the actuator.

(1) Estimation of the current posture. When a stimulation is decided to be sent, the first step is to recognize the current posture of the subject. All the data the algorithm has are the current pressure map and the 3 pre-acquired pressures maps that correspond to the left, central and right Reference Postures. Estimating what is the current posture of the subject consists in calculating the distance between the current pressures map and the pre-recorded ones. This distance is the Manhattan distance of the Euclidean space:

$$
d(A, B)=\sum_{\text {sensors } s}|A(s)-B(s)|
$$

In the above formula, $\mathrm{A}$ is the current pressure map, B is one of the stored Reference Postures pressure maps and $\mathrm{s}$ are the sensors indices.

Three distances are thus calculated (with B corresponding to RP_L, RP_C and RP_R) and the algorithm assumes that the current posture is the one for which the distance is the smallest. In the example presented in Fig. 3, the central posture is considered as the current one.

(2) Calculation of the posture that is the most suitable to reduce cumulated overpressures. The 


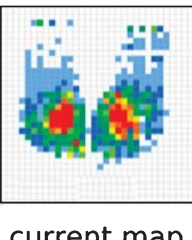

current map

current posture

(lowest d score)

target posture

(highest wd score)

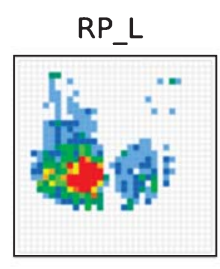

$8 K$

$206 K$

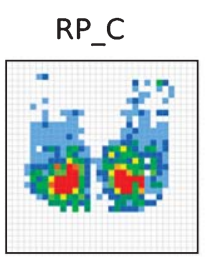

$2 \mathrm{~K}$

$82 K$

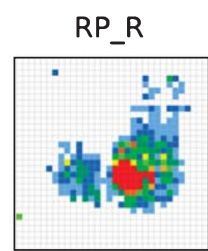

$11 K$

$257 K$

Fig. 3. Algorithm decision. When a biofeedback is decided, the algorithm estimates the current posture of the subject by calculating a distance between the current map and the three pre-acquired maps (in this example, the current posture is the central one). Next, the algorithm decides which one of the other postures is the most suitable by calculating another distance (in this example, the target posture is the right one). Finally, the opposite side of the calculated postural mobilization (towards right here) is activated (left activation).

next step is to "drive" the subject towards the best posture. As we want this posture to reduce the cumulated overpressures, the algorithm will calculate another score, providing more weights to previously cumulated high pressures and to their decrease. This is done by the following distance formula $w d$ :

$w d(A, B)=\sum_{\text {sensors } s} t(A(s)) \cdot(A(s)-B(s))$

where $\mathrm{t}(\mathrm{A}(\mathrm{s}))=\max (\mathrm{A}(\mathrm{s})-100,0)$ aims at focusing on pressure sensors values that are greater than $100 \mathrm{mmHg}$.

The $w d$ score is calculated between the current map A and the two pre-acquired maps B that do not correspond to the current posture. This time, the posture for which the score is maximal (indicating a maximal decrease of the high pressures) is considered as the target posture towards which the subject has to be guided.

In the example presented in Fig. 3, the target posture corresponds to a chest displacement to the right.

(3) Activation of the actuator. Once the current and target postures are determined, the algorithm can easily deduce in which direction the subject has to move (right direction in our example (Fig. 3)). Since we wanted the biofeedback signal to be similar to the paresthesic pain signal, the activated side was the one that was supposed to be over-pressurized. In the example illustrated on Fig. 3, the left side of the actuator was activated.

\subsubsection{Data analysis}

At a first step, two quantitative variables will be analysed to assess the efficiency of the perceptual supplementation device with the different sensory modalities.

(1) The first variable is the correctness of the task realisation. To do this, we will notice, for each subject, (i) the number of stimulations for which he/she reacted correctly (i.e. the posture after the signal is the target one), (ii) the number for which he/she did not react (posture after the signal is the same as the beginning, assuming that the signal was not perceived at all or neglected) and (iii) the number for which he/she had an incorrect reaction (i.e. the posture after the stimulation is neither the target one nor the same as the beginning, assuming that the signal was misunderstood).

(2) The second variable is a measurement of the potential decrease of high pressures. We consider here only the sensors for which the values before the signal were over a certain threshold (this threshold was empirically chosen at 
$100 \mathrm{mmHg}$, after discussions with clinicians and observations of typical pressure maps), because the higher the pressures are, the more dangerous they are. These sensors define a risky region named "Arisk". The variable we propose to calculate here is the variation of the volume of pressures in Arisk (directly proportional to the sum of the values of the sensors forming Arisk) before the biofeedback versus after the biofeedback.

In addition, two qualitative variables were computed in order to estimate the acceptability of each biofeedback. Subjects were asked to quantify the ergonomics of the different biofeedbacks using a questionnaire by grading the following properties:

(1) Comfort. First, subjects were asked to provide a grade (from 0 to 10) for the comfort of each biofeedback. Comfort gathers the notions of physical comfort (discomfort of the material components of the actuator, discomfort of the stimulation...) and the psychological feeling (by imagining using this device in day-life activities);

(2) Disturbances in relation to the external perceptions. Subjects were asked to provide a grade (from 0 to 10 ) concerning the eventual interferences between the stimulations of the device and the external perceptions (i.e. were the stimulations of the device disturbing the sensory perceptions from the environment and viceversa?).

\section{Results}

Figure 4 shows the results for the first quantitative variable. We can see that all the stimulations in the control session have led to non-reactions while the large majority of stimulations in the other conditions have been correctly interpreted (between $92 \%$ for the tactile modality to $100 \%$ for the visual and auditory ones).

Figure 5 plots the results for the second variable. We observe that overpressures reduction were higher in every modality compared to the control condition.

Table 1 shows the results for the questionnaire about the ergonomics. We can see that the auditory modality was granted by 6.61 in term of comfort and 7.47 in term of disturbance; the visual one was granted by 8.22 in term of comfort and 7.22 in term of disturbance; and

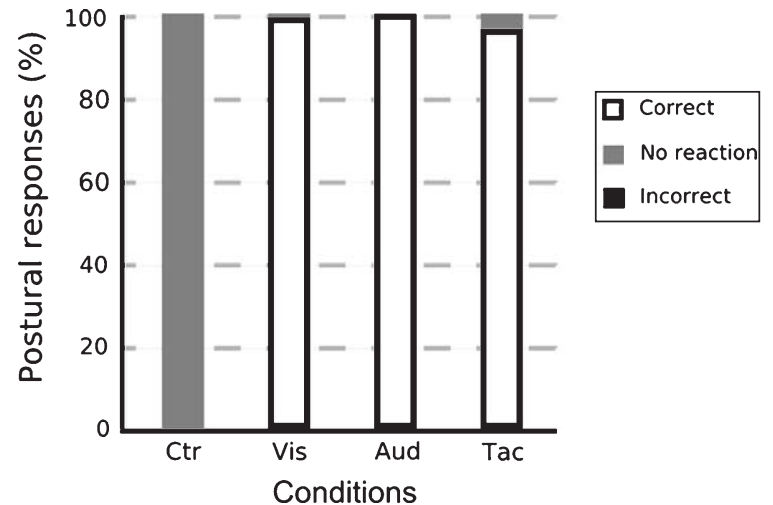

Fig. 4. Postural responses in each sensory modality. Ctr, Visu, Aud and Tac are respectively for Control, Visual, Auditory an Tactile conditions.

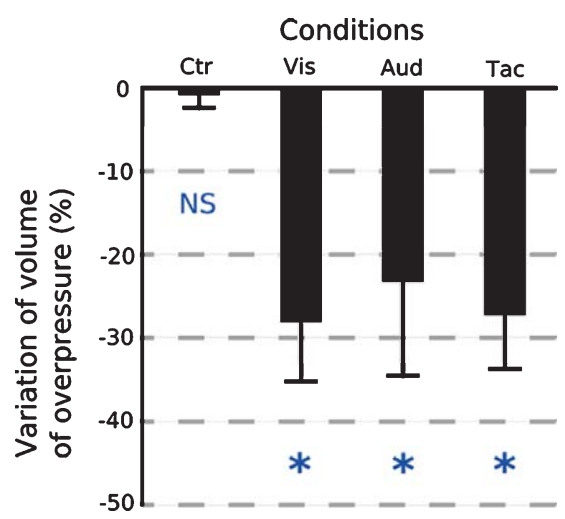

Fig. 5. Reduction of overpressures after the biofeedback in each sensory modality.

Table 1

Results of the questionnaire (mean values) about the acceptability of each sensory modality

\begin{tabular}{lccc}
\hline & $\begin{array}{c}\text { Auditory } \\
\text { modality }\end{array}$ & $\begin{array}{c}\text { Visual } \\
\text { modality }\end{array}$ & $\begin{array}{c}\text { Electrotactile } \\
\text { modality }\end{array}$ \\
\hline Comfort & 6.61 & 8.22 & 4.44 \\
Disturbance & 7.47 & 7.22 & 7.72 \\
\hline
\end{tabular}

finally the lingual electrotactile one was granted by 4.44 in term of comfort and 7.72 in term of disturbance.

\section{Discussion}

The first aim of this study was to evaluate the efficiency of the lingual electrotactile modality as a 
biofeedback device for pressure sore prevention, comparatively to more usual sensory modalities such as vision and audition.

First, the results show that all sensory modalities seem to be efficient, in term of perception. The stimulations are correctly perceived and interpreted. Despite the fact that the worst score is the one with the tactile feedback, this score remains quite good (92\%) and the stimulations that are not correctly perceived can be detected by the algorithm and taken into consideration immediately.

Moreover, the results concerning the reductions of overpressures indicate that, whatever is the sensory modality, the mean reduction is globally identical. Results show indeed decreases of $23 \%$ for the auditory modality, $28 \%$ for the visual one and $27 \%$ for the electrotactile one. These scores are all significantly different from the control condition, evidencing a certain efficiency of the system, whatever the modality is. Moreover, these scores are not significantly different from each other, indicating a globally similar efficiency.

The second aim of this study was to assess the ergonomics and acceptability of the actuator part of the perceptual supplementation device. The electrotactile modality obtains here a very low grade. Subjects said that, in spite of the efforts made in the design of a miniaturized and embedded TDU, they would not accept such a device in mouth during a day-life activity. This could be explained by the size of the device, still large and uncomfortable to be kept inside the mouth or by the unusual type of stimulation that may be disturbing for subjects. We believe that recent advances in technology could allow to greatly miniaturize this device, and a perspective of research is to improve this ergonomics.

However, in a perspective of a fast supply for a daylife use, the actuator has to be re-designed. Auditory and visual modalities received very good notations of comfort, but the results presented in Table 1 in term of disturbance with the environment perceptions are worst than those obtained with the electrotactile modality. Moreover, it seems difficult to imagine a purely visual or auditory actuator for a day-life use. An always visible screen or a bell may not be accepted by users since such disposals are invasive towards the surrounding people.
That is one of the reasons why we believe that a tactile modality is unavoidable. Since the stimulations of our system are not continuous, a bimodal actuator may be appropriate. Like a cellular phone, this one would consist of a vibrotactile alert and a screen that the user could watch afterwards. This could take shape of a phone or a watch. Some studies about such devices are currently running in our laboratory.

\section{Acknowledgments}

The authors would like to thank subject volunteers and the reviewers for helpful comments and suggestions. This research was granted by the Garches Fundation, the AXA Research Fund, the company IDS SA and the cluster HNV. The company Vista Medical is acknowledged for supplying the FSA pressure data acquisition system.

\section{References}

[1] http://www.npuap.org.

[2] D. Colin, Le poids socio-économique des escarres. Les escarres, actes des 13emes entretiens de l'institut Garches, Lortat-Jacob A et Colin D (2000), 77-81.

[3] C. Kauer and P. Fontet, Escarres ischiatiques: Techniques et classifications. Les escarres, actes des 13emes entretiens de l'institut Garches, Lortat-Jacob A et Colin D (2000), 77-81.

[4] P. Bach-y Rita, C.C. Collins, F.A. Saunders, B. White and L. Scadden, Vision substitution by tactile image projection, Nature 221 (1969), 963-964.

[5] O. Chenu, N. Vuillerme, J. Demongeot and Y. Payan, A wireless lingual feedback device to reduce overpressures in seated posture : A feasibility study, PLoS ONE 4 n. 10 (2009), e7550.

[6] P. Bach-y Rita, K.A. Kaczmarek, M.E. Tyler and J. GarciaLara, Form perception with a 49-point electrotactile stimulus array on the tongue: A technical note, Journal of Rehabilitation Research and Development 35 n.4 (1998), 427.

[7] N. Vuillerme, N. Pinsault, O. Chenu, J. Demongeot, Y. Payan, et al., Sensory supplementation system based on electrotactile tongue biofeedback of head position for balance control, Neuroscience Letters 431 (2007), 206-210.

[8] N. Vuillerme, N. Pinsault, O. Chenu, M. Boisgontier, J. Demongeot, et al., How a plantar pressure-based, tongue-placed tactile biofeedback modifies postural control mechanisms during quiet standing, Experimental Brain Research 181 (2007), 547-554.

[9] F. Robineau, N. Vuillerme, J.P. Orliaguet and Y. Payan, Tongue liminary threshold identification to electrotactile stimulation. Proceedings of the 4th international conference on enactive interfaces, Enactive'07, Arxiv preprint arXiv:0711.4768, (2007), pp. 233-236. 

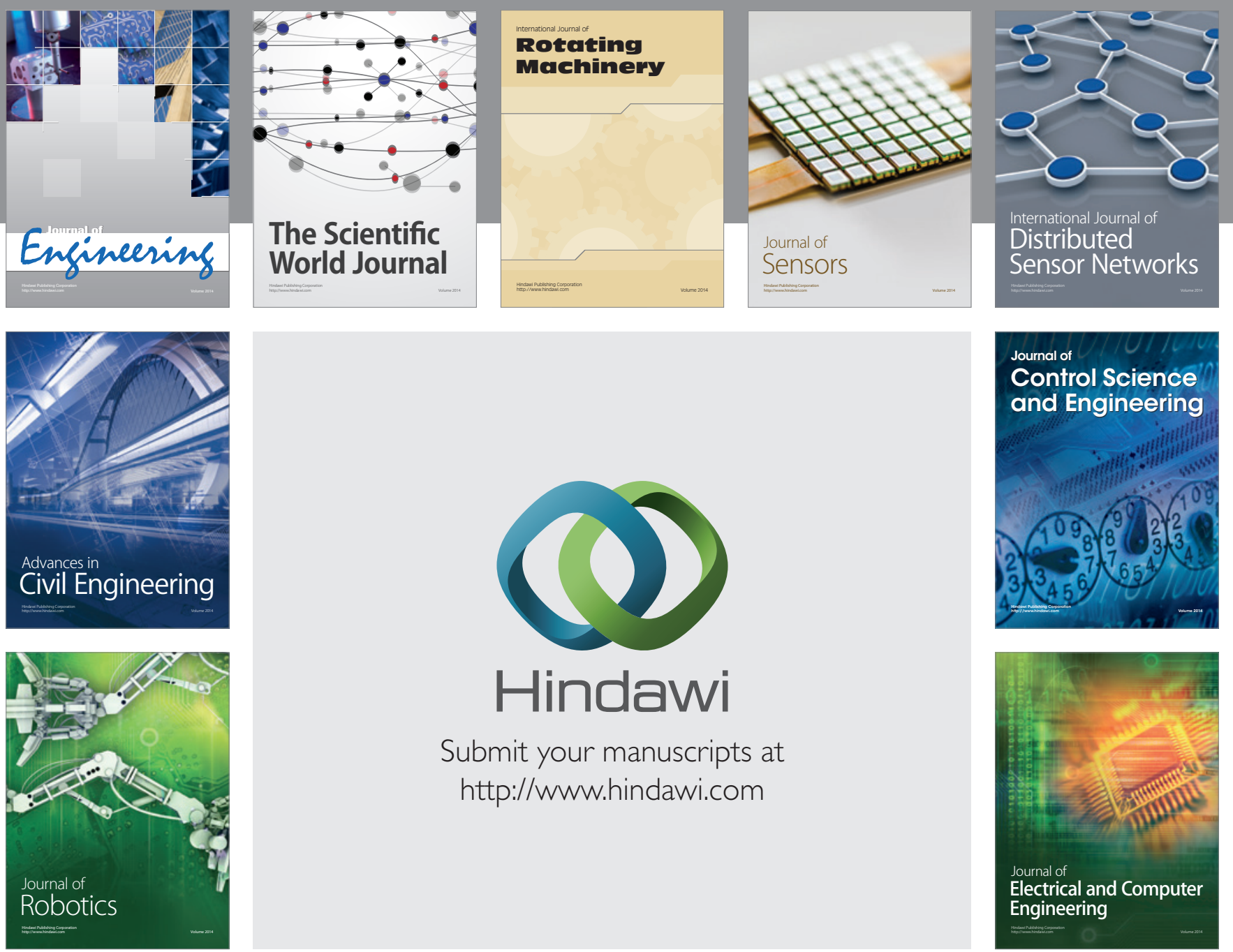

Submit your manuscripts at

http://www.hindawi.com
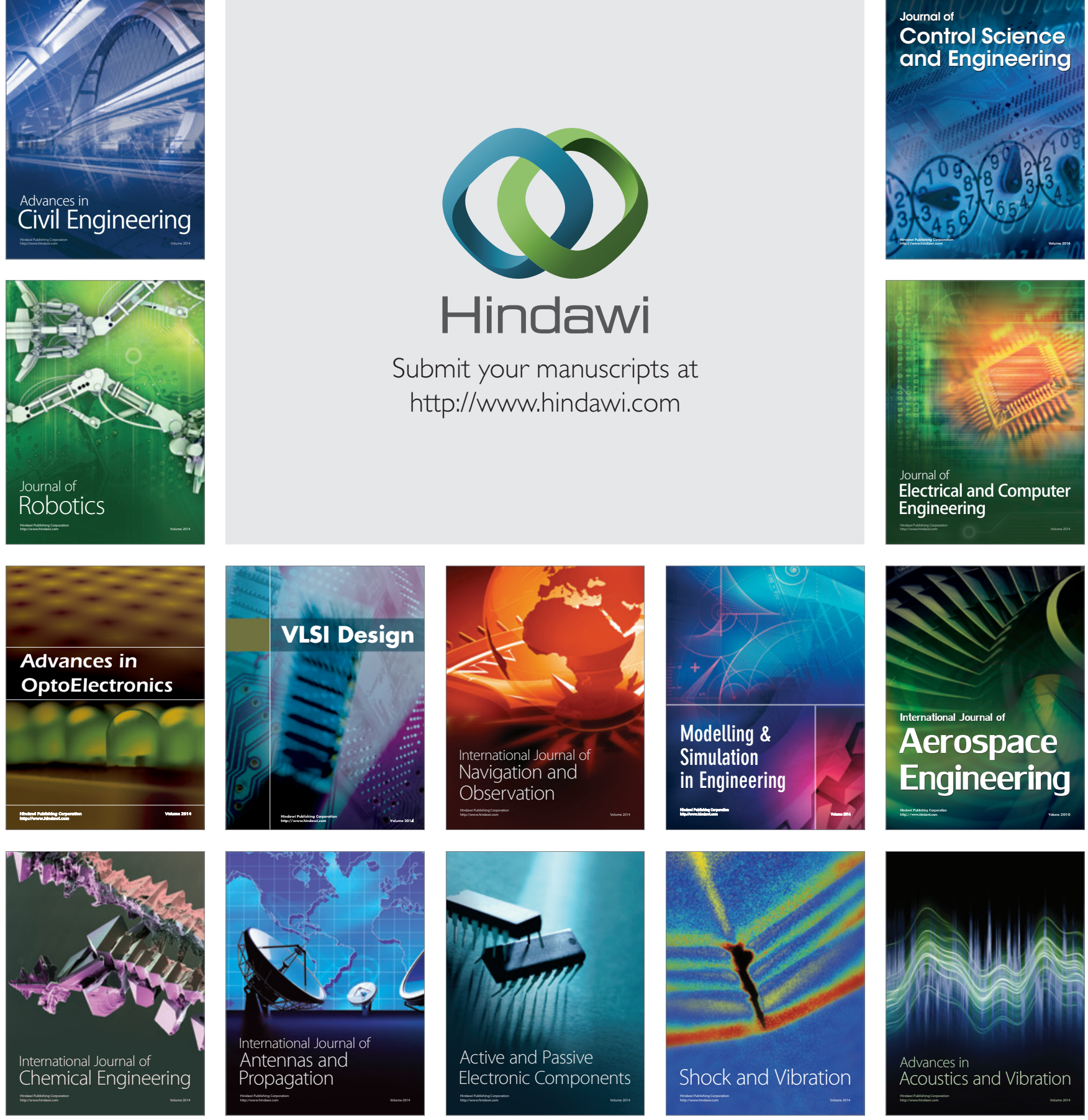